EPJ Web of Conferences 108, 02010 (2016)

DOI: $10.1051 /$ epjconf/201610802010

C) Owned by the authors, published by EDP Sciences, 2016

\title{
Algorithm for Solving an Optimization Problem for the Temperature Distribution on a Plate
}

\author{
A. Ayriyan ${ }^{1, a}$, E.E. Donets ${ }^{1}$, H. Grigorian ${ }^{1,2}$, N. Kolkovska ${ }^{3}$, and A. Lebedev ${ }^{1,4}$ \\ ${ }^{1}$ Joint Institute for Nuclear Research, Joliot-Curie 6, 141980 Dubna, Moscow Region, Russia \\ ${ }^{2}$ Yerevan State University, Alek Manyukyan 1, 0025 Yerevan, Republic of Armenia \\ ${ }^{3}$ Institute of Mathematics and Informatics of BAS, Acad. Georgi Bonchev 8, 1113 Sofia, Bulgaria \\ ${ }^{4}$ GSI Helmholtzzentrum für Schwerionenforschung, Planckstraße 1, 64291 Darmstadt, Germany
}

\begin{abstract}
The work describes the maximization problem regarding the heating of an area on the surface of a thin plate within a given temperature range. The solution of the problem is applied to ion injectors. The given temperature range corresponds to the required pressure of a saturated gas comprising evaporated atoms of the plate material. In order to find the solution, a one-parameter optimization problem was formulated and implemented leading to the optimization of the plate specific geometry. It was shown that a heated area can be increased up to $23.5 \%$ in comparison with a regular rectangle form of a given plate configuration.
\end{abstract}

\section{Introduction}

The work describes the maximization problem regarding heating of an area on the surface of a thin plate within a given temperature range. The plate serves for injecting the working species (atoms of the plate material evaporated from its surface) into the working space of an ion source [1]. The plate is heated by the flux of the electric current passing through it. The injection starts when the temperature reaches the required value depending on the material of the plate. The temperature range and the working area of the surface respectively are defined by the required pressure of a saturated vapor above the surface of the plate.

In this work a model of the plate and a one-parameter variation of its geometry are discussed (see figure 1). In the existing technical device the plate has a rectangular form (figure 1a). In order to maximize the working area on its surface we suggested to change the geometry in the following way. A new shape has been derived from the regular one by the removal of rectangular parts from the corners. Thus rectangular wing-like structures (further referenced as wings) appear on the both sides of the plate (figure 1b) with their length being left as a free parameter. Our simulations have shown that the working area of the plate has approximately a rectangular shape for the used set of parameters. The shape of the plate has been optimized by varying the length of these wings in order to reach a maximum working area. The procedure has been applied under the condition that the highest temperature has to be significantly less than the melting temperature of the plate material.

\footnotetext{
ae-mail: ayriyan@jinr.ru
} 


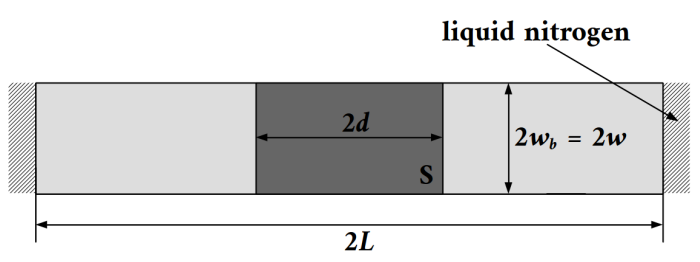

(a) Sketch of the existing plate geometry.

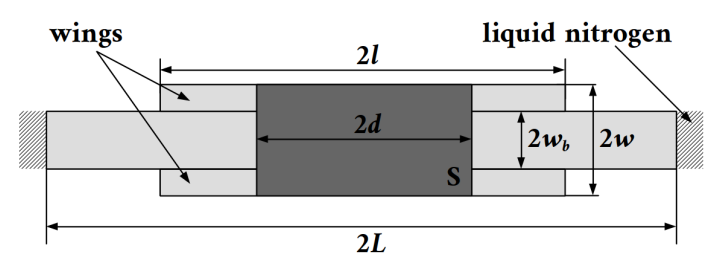

(b) Sketch of the suggested geometry.

Figure 1: The geometry of the plate. Here $2 L$ is the length of the plate, $2 w$ is the width of the plate, $2 l$ is the length of the wings, $2 w_{b}$ is the width without wings, $S$ is the working area of the plate surface heated to the required temperatures, $2 d$ is the length of the working area. Left and right sides are connected to the temperature terminal.

\section{Main Equation and Conditions}

The stationary temperature distribution in the plate is modelled by the equation [2]:

$$
\frac{\partial}{\partial x}\left(\lambda(T) \frac{\partial T}{\partial x}\right)+\frac{\partial}{\partial z}\left(\lambda(T) \frac{\partial T}{\partial z}\right)+\frac{I^{2} \chi(T)}{S_{\mathrm{C}}^{2}}=0,
$$

where the thermal conductivity $\lambda(T)$ and the resistivity $\chi(T)$ depend non-linearly on a sought-after function (temperature), $S_{\mathrm{C}}$ is the cross-sectional area, and $I$ is the electric current.

Due to the symmetry at the middle of the plate along the $x$-and $z$-axes, solving the problem on a quarter of the full domain is sufficient (see figure 2):

The boundary conditions can be taken as following:

$$
\begin{cases}\frac{\partial T}{\partial \mathbf{n}}=0 & \text { if }(x, z) \in \partial \Omega \backslash\{(x, z) \mid z=L\}, \\ T=T_{0} & \text { if }(x, z) \in\{(x, z) \mid z=L\} .\end{cases}
$$

The right side of the plate is connected to the temperature terminal with $T_{0}=78 \mathrm{~K}$. At the boundaries of the

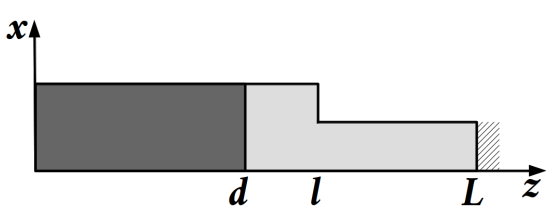

Figure 2: $\Omega$-domain of simulations. domain the temperature gradient vanishes: along the $x$ - and $z$-axes because of the symmetry and at the other points because the plate is placed inside the vacuum chamber.

The problem (1)-(2) can be solved by various methods. We have chosen the method deriving the solution of the elliptic equation (1) as a stationary solution of the parabolic one (3):

$$
\rho(T) c_{V}(T) \frac{\partial T}{\partial t}=\frac{\partial}{\partial x}\left(\lambda(T) \frac{\partial T}{\partial x}\right)+\frac{\partial}{\partial z}\left(\lambda(T) \frac{\partial T}{\partial z}\right)+I^{2} X(T),
$$

here the density is $\rho(T)$, the heat capacity is $c_{V}(T)$, and $X(T)=\chi(T) / S_{\mathrm{C}}^{2}$. The solution of the elliptic equation (1) is the stationary solution of the heat equation (3). In order to solve the equation (3) we have assumed the initial condition:

$$
T=T_{0} \quad \forall(x, z) \in \Omega .
$$

\section{Formulation of the Optimization Problem}

The optimization problem has been formulated to maximize the working area. The temperature range event from $T_{\text {low }}=678 \mathrm{~K}$ to $T_{\text {high }}=778 \mathrm{~K}$. $T_{\text {high }}$ is significantly less than the melting point of the plate material. The maximum temperature constraint is applied to the hottest point on the surface of the plate which is located at its center due to the plate homogeneity and symmetry. 
The size of the working area is proportional to $d$. Thus the maximum of the working area is reached for the maximum of $d$. Note that the width of the plate, $2 w$, is constant. It reaches the maximum possible value following from the technical requirements. The relation $w_{b} / w$ is fixed. Being dependent on the semilength of the wings $l$ (figure 3 ) for the given temperature $T_{\text {low }}, d$ is the solution of the following equation for $z$ :

$$
T(x=0, z ; l)-T_{\text {low }}=0,
$$

when we choose the source coefficient $I$ (electrical current) corresponding to the maximum temperature constraint.

Therefore, the maximum of the working surface area $S$ corresponds to the maximum of $d(l)$ :

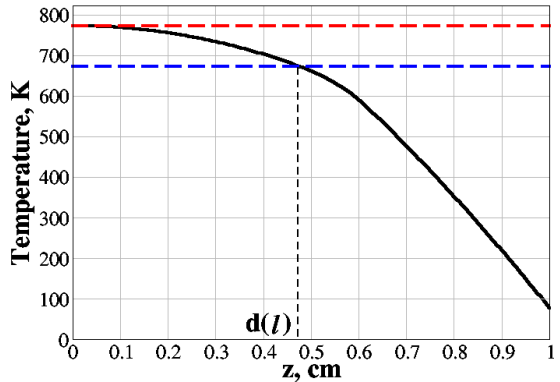

Figure 3: A typical temperature profile versus $z$ for different lengths of the wings at $x=0$. The dashed lines correspond to $T_{\text {low }}=678 \mathrm{~K}$ and $T_{\text {high }}=778 \mathrm{~K}$.

$$
d\left(l^{*}\right)=\max _{0 \leq l \leq L}|d(l)| .
$$

The solution of the optimization problem requires repeated solutions of the heat conduction problem (2)-(4).

\section{Numerical Algorithms}

\subsection{Solving the Direct Problem}

The finite difference method has been chosen to solve the heat conduction problem (2)-(4):

$$
\begin{gathered}
\rho_{i, j} c_{V i, j} \frac{\widehat{T}_{i, j}-T_{i, j}}{\tau}=\Lambda_{X}\left(\widehat{T}_{i, j}\right)+\Lambda_{Z}\left(T_{i, j}\right)+I^{2} X_{i, j}, \\
\left\{\begin{array}{l}
\Lambda_{X}\left[\widehat{T}_{i, j}\right]=\frac{1}{\hbar_{i}}\left[\lambda_{i+\frac{1}{2}, j} \frac{\widehat{T}_{i+1, j}-\widehat{T}_{i, j}}{h_{i+1}}-\lambda_{i-\frac{1}{2}, j} \frac{\widehat{T}_{i, j}-\widehat{T}_{i-1, j}}{h_{i}}\right], \\
\Lambda_{z}\left[T_{i, j}\right]=\frac{1}{\eta_{j}}\left[\lambda_{i, j+\frac{1}{2}} \frac{T_{i, j+1}-T_{i, j}}{\eta_{j+1}}-\lambda_{i, j-\frac{1}{2}} \frac{T_{i, j}-T_{i, j-1}}{\eta_{j}}\right],
\end{array}\right.
\end{gathered}
$$

where $i=1 \ldots N_{j}-1, j=1 \ldots M_{i}-1, h_{i}=x_{i}-x_{i-1}, \eta_{j}=z_{j}-z_{j-1}, \hbar_{i}=\left(h_{i+1}+h_{i}\right) / 2$, $\eta_{j}=\left(\eta_{j+1}+\eta_{j}\right) / 2, T_{i, j}=T\left(x_{i}, z_{j}, t_{k}\right), \widehat{T}_{i, j}=T\left(x_{i}, z_{j}, t_{k+1}\right), c_{V i, j}=c_{V}\left(T_{i, j}\right), X_{i, j}=X\left(T_{i, j}\right)$, $\lambda_{i \pm \frac{1}{2}, j}=\lambda\left(\left(T_{i, j}+T_{i \pm 1, j}\right) / 2\right), \lambda_{i, j \pm \frac{1}{2}}=\lambda\left(\left(T_{i, j}+T_{i, j \pm 1}\right) / 2\right)$.

The difference scheme (7)-(8) has been solved by the Thomas algorithm [3, 4]:

$$
\alpha_{i}=\frac{-C_{i}}{B_{i}+A_{i} \alpha_{i-1}}, \quad \beta_{i}=\frac{F_{i}-A_{i} \beta_{i-1}}{B_{i}+A_{i} \alpha_{i-1}}, \quad \widehat{T}_{i, j}=\alpha_{i} \widehat{T}_{i+1, j}+\beta_{i} .
$$

The coefficients $A_{i}, B_{i}, C_{i}$, and $F_{i}$ are defined from the difference equation (7):

$$
\left\{\begin{aligned}
A_{i} & =-\frac{\lambda_{i-\frac{1}{2}, j}}{\hbar_{i} h_{i}}, \\
B_{i} & =\frac{1}{\hbar_{i}}\left[\frac{\lambda_{i-\frac{1}{2}, j}}{h_{i}}+\frac{\lambda_{i+\frac{1}{2}, j}}{h_{i+1}}\right]+\frac{\rho_{i, j} c_{V i, j}}{\tau}, \\
C_{i} & =-\frac{\lambda_{i+\frac{1}{2}, j}}{\hbar_{i} h_{i+1}} \\
F_{i} & =\frac{\rho_{i, j} c_{V i, j}}{\tau} T_{i, j}+\Lambda_{z}\left[T_{i, j}\right]+I^{2} X_{i, j} .
\end{aligned}\right.
$$


The boundary conditions (2) are approximated in the Thomas algorithm as follows:

$$
\begin{gathered}
\alpha_{0}=\frac{2 \lambda_{0, j} \tau}{h_{1}^{2} \rho_{0, j} c_{V 0, j}+2 \lambda_{0, j} \tau}, \quad \beta_{0}=\frac{\rho_{0, j} c_{V 0, j} T_{0, j}+\tau\left(\Lambda_{z}\left[T_{0, j}\right]+I^{2} X_{0, j}\right)}{h_{1}^{2} \rho_{0, j} c_{V 0, j}+2 \lambda_{0, j} \tau} h_{1}^{2}, \\
\widehat{T}_{N_{j}, j}=\frac{\left[4-\alpha_{N_{j}-2}\right] \beta_{N_{j}-1}-\beta_{N_{j}-2}}{3-\alpha_{N_{j}-1}\left[4-\alpha_{N_{j}-2}\right]} .
\end{gathered}
$$

The initial condition corresponding to (4) is $T_{i, j}=T_{0} \forall i, j$.

The difference scheme (7)-(12) gives a second order approximation of the heat conduction problem (2)-(4) with respect to the spatial steps while first order with respect to time-step. The scheme is unconditionally stable relating to spatial step $h_{i}$ and conditionally stable relating to $\eta_{j}$ [5]:

$$
\tau \leq \frac{\min \left|\eta_{j}^{2}\right|}{2} \cdot \min \left|\frac{\rho(T) c_{V}(T)}{\lambda(T)}\right| .
$$

\subsection{Algorithm for Solving the Optimization Problem}

The algorithm comprises the following steps: 1) the length of the wings $l \in[0, L]$ is sampled with a number $N_{\text {wings }} ; 2$ ) for each $l_{i}$ the source problem is solved and $d\left(l_{i}\right)$ is calculated; 3 ) the value of $l^{*}$ is found according to the maximum value of $d$.

The value of the electric current $I$ varies in the range $\left[0, I_{\max }=500 \mathrm{~mA}\right]$. If $I$ reaches $I_{\max }$, the temperature at the central point of the plate exceeds the maximum temperature constraint for all $l \in[0, L]$. In order to find the unique solution existing within the defined range of $I$, the bisection method is used.

\subsection{Parallel Algorithm}

A parallel algorithm for solving the optimization problem was implemented with the usage of Message Passing Interface (MPI) [6]. The main loop of the algorithm 1 (line 2) was parallelized. For each $l_{i}$ the source problem is solved by a separate parallel process. The parallel algorithm may be executed up to $N_{\text {wings }}$ times faster in comparison with the sequential one.

\section{Results}

Numerical results are reported for a plate made of Thulium. Generally, the algorithm has been developed for temperature values depending on the thermal coefficients, but for the present simulations the thermal coefficients $[7,8]$ are assumed to be constant for the considered temperature range: the conductivity is $\lambda=0.169 \mathrm{~J} /(\mathrm{cm} \mathrm{s} \mathrm{K})$, the specific heat is $c_{V}=0.16 \mathrm{~J} /(\mathrm{g} \mathrm{K})$, the density is $\rho=9.33 \mathrm{~g} / \mathrm{cm}^{3}$, and the ratio of the resistivity to the square of the cross-section area is considered to be the same throughout the whole plate $\chi / S_{\mathrm{C}}^{2}=10^{3} \times 10^{3} \mathrm{Ohm} / \mathrm{cm}^{3}$. The fixed size of the plate was $L=1 \mathrm{~cm}$, $w_{b}=0.05 \mathrm{~cm}$ and $w=0.125 \mathrm{~cm}$ (figure 1b). The sampling number $N_{\text {wings }}=10$.

One can see from the figure 4a that the temperature profile is sensitive to the length of the wings. The function $d(l)$ and the temperature field for $l^{*}$ are shown in figures $4 \mathrm{~b}$ and $4 \mathrm{c}$ respectively. The maximum $d$ corresponds to $l^{*}=0.6 \mathrm{~cm}$.

Fig. 5 illustrates the performance of the parallel algorithm depending on the number of CPUs $-n_{p}$. The calculations have been carried out on the HybriLIT heterogenius cluster (CPU - Intel Xeon E52695). Fig. 5a shows the calculation time $t_{n_{p}}$, Fig. $5 \mathrm{~b}-$ the speedup of calculations $t_{1} / t_{n_{p}}$, and Fig. 5c - the efficiency of the parallelization $t_{1} /\left(n_{p} \times t_{n_{p}}\right)$. 


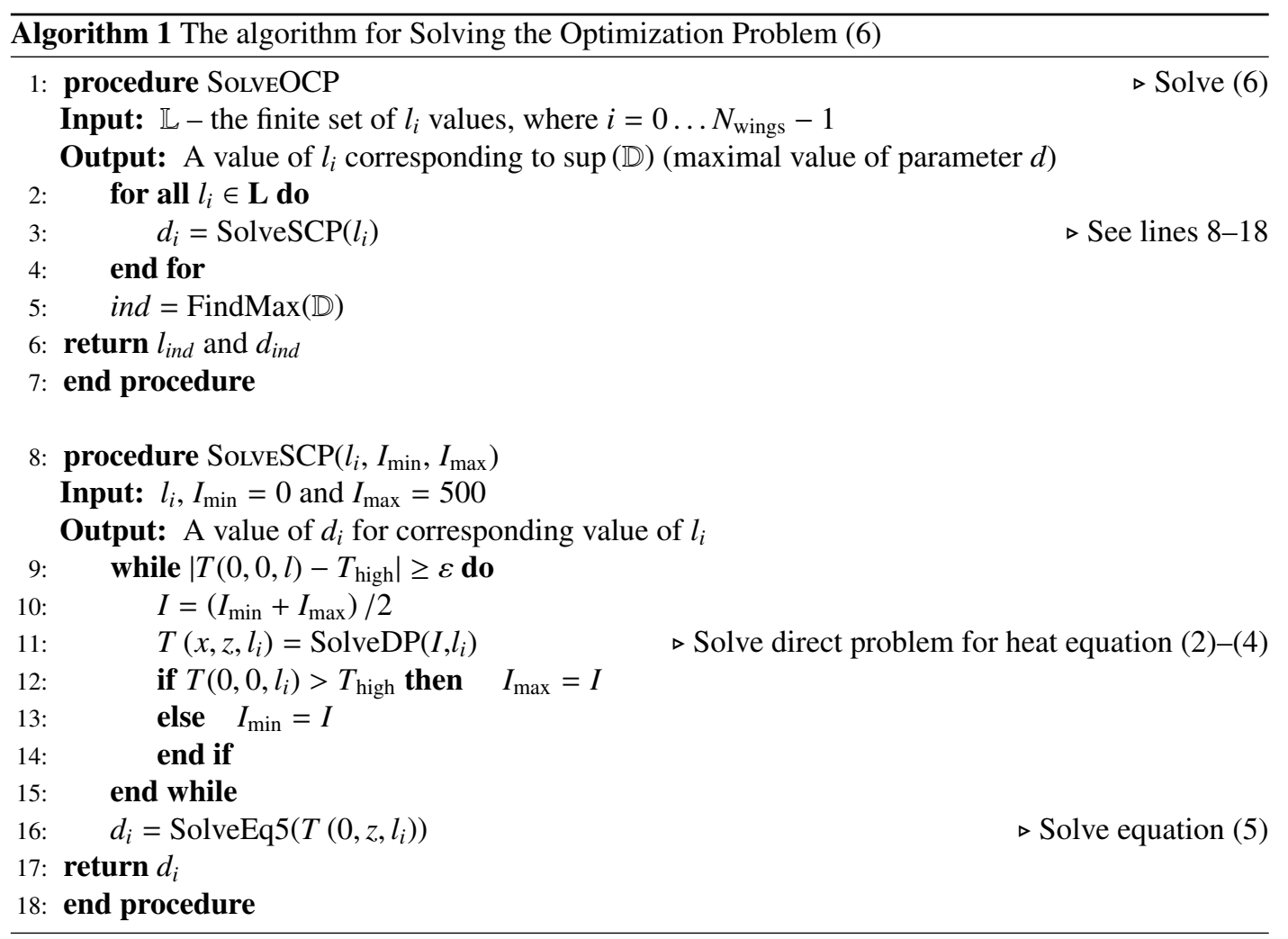



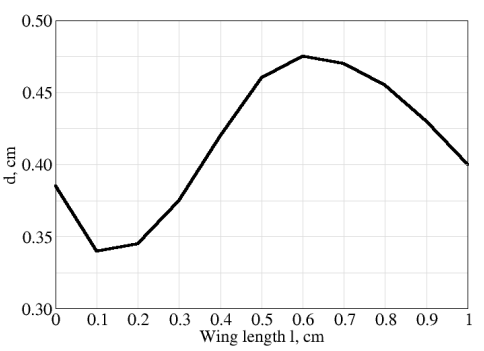

(a) Temperature profiles for different lengths of wings (b) Parameter $d$ for different lengths of wings

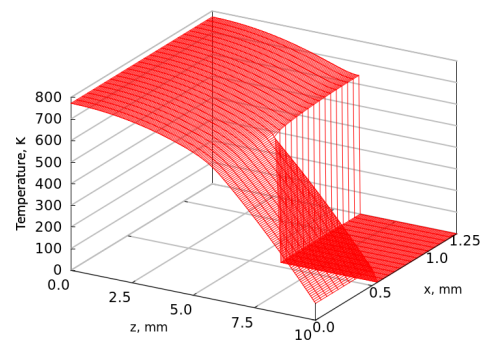

(c) Temperature field in quota of the plate

Figure 4: Results of solving the source problem as a function of $l$

\section{Summary and Conclusion}

In order to maximize the working area we have suggested changing the plate geometry. The new shape has been derived from the regular one by removal of rectangular parts from its corners. The simulations have been carried out in order to find the optimal plate geometry. For that purpose an algorithm for solving the one-parameter optimization problem has been developed and its parallel version has been implemented. 


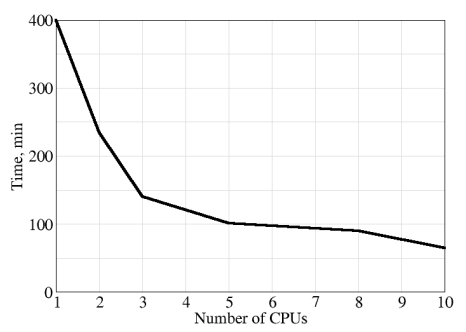

(a) Calculation time $t_{n_{p}}$ in $\min$

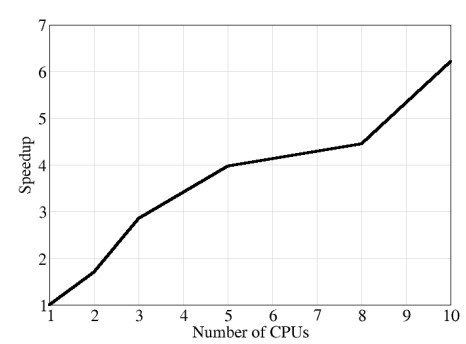

(b) Speedup: $t_{1} / t_{n_{p}}$

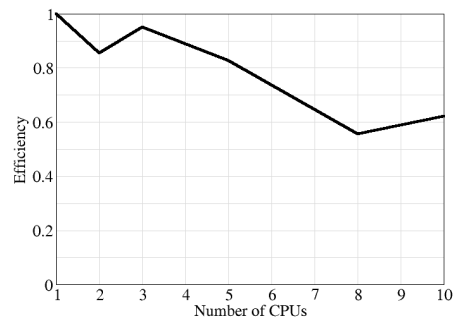

(c) Efficiency: $t_{1} /\left(n_{p} \times t_{n_{p}}\right)$

Figure 5: Results of the parallel algorithm: time, speedup and efficiency of calculations in dependence of number of processors $-n_{p}$

The optimal geometry of the plate has been found $\left(l^{*}=0.6 \mathrm{~cm}\right)$. This allowed an increase of the working area up to $23.5 \%$ in comparison with the one of the regular plate. The value of the electric current corresponding to the maximum working area has been found to be equal to $I=385 \mathrm{~mA}$.

\section{Acknowledgements}

The study is partially supported by RFBR according to the projects No. 14-01-00628 and No. 14-01-31227. Authors thank Edik Ayryan (JINR) for permanent interest in this research and worthwhile discussions, and S. Lebe$\operatorname{dev}$ (JINR \& Giessen University) for helpful remarks.

\section{References}

[1] D.E. Donets, E.D. Donets, E.E Donets, V.V Salnikov, V.B. Shutov, Journal of Instrumentation 5, C09001 (2010)

[2] A.A. Samarskii, P.N. Vabishchevich, Computational Heat Transfer, (Volume 1, John Wiley \& Sons Ltd., Chichester, England, 1995)

[3] L.H. Thomas, Elliptic Problems in Linear Differential Equations over a Network (Watson Sci. Comput. Lab Report, Columbia University, New York, 1949)

[4] W.H. Press, S.A. Teukolsky, W.T. Vetterling, B.P. Flannery, Numerical Recipes, third ed. (Cambridge University Press, New York, 2007)

[5] N.N. Yanenko, Fractional step methods for solution of multidimensional problems of mathematical physics (Nauka, Moscow, 1967) (in russian)

[6] The Open MPI developer community, http://www.open-mpi.org/ (11/06/2015)

[7] I.S. Grigoreva, E.Z. Meylihova, Physical quantities. Handbook (Energoatomizdat, Moscow, 1981)

[8] Thulium: physical properties, http://www.webelements.com/thulium/physics.html (05/04/2015) 